\title{
CASE REPORT \\ CORONARY ARTERY FISTULA IN ELDERLY PATIENT WITH ACUTE CORONARY SYNDROME: A CASE REPORT
}

\author{
Quan Vo', Hong Vu², Thanh Dinh ${ }^{2}$ \\ ${ }^{1}$ University of Medicine and Pharmacy, Ho Chi Minh City, Ho Chi Minh, Vietnam, ${ }^{2}$ Binh Dan Hospital, Ho Chi Minh City, Ho Chi \\ Minh, Vietnam
}

Coronary artery fistulas are congenital or acquired abnormality connections between coronary arteries and other structures. Most patients with coronary fistulas are asymptomatic. An 80year-old man complained of exercise-induced chest pain. A coronary angiogram on PCI and CTA confirmed stenosis on LAD and a large fistula between the coronary artery and the anterior side of the main pulmonary artery. A transcatheter procedure was successfully conducted. In patients with symptomatic fistula or cases with a complication, cardiac intervention is recommended as the primary treatment. Percutaneous catheterization is the preferred treatment. Keywords: ischemic heart disease, coronary fistula, angiography

Citation: Vo Q, Vu H, Thanh Dinh T. Coronary Artery Fistula in Elderly Patient with Acute Coronary Syndrome: A Case Report. Pak Heart J. 2021;54(02):198-200. DOI: https://doi.org/10.47144/phj.v54i2.2099

\section{INTRODUCTION}

Coronary artery fistula is an uncommon coronary malformation estimated to account for $0.05 \%$ of congenital cardiac anomalies in the general population. In 1865, Krause described the first case of a coronary fistula between coronary arteries and cardiac chambers. Since then, many cases have been reported worldwide. Patients with coronary artery fistulas have varied morphological appearances and symptoms when the lesion is discovered. The most distinct origins of coronary fistula are the right coronary artery (RCA) and left anterior descending artery (LAD). Their clinical importance is usually due to the development of complications, including heart failure, thrombosis, thromboembolic events, endocarditis, arrhythmias, and ruptured aneurysm. We present a recent case about an elderly patient hospitalized with signs of acute coronary syndrome accompanied by coronary artery fistula. The critical information was described with the aim of contributing to the comprehensive care plan for this rare entity.

\section{CASE REPORT}

An 80-year-old retired male patient was presented to the Emergency Department with a history of exercise-induced chest pain and dyspnea constantly aggravated over months. He was a healthy person and his medical history revealed no cardiac disease, although he had smoked one pack of cigarettes per day for 30 years. The annual routine cardiology investigation found no abnormalities.

On recent presentation, he was afebrile with a pulse rate, $102 \mathrm{bpm}$, blood pressure, $113 / 53 \mathrm{mmHg}$, respiration rate, 26 breaths/min, and oxygen saturation, $92 \%$ on ambient air. Physical examination revealed no other abnormal signs. An electrocardiogram at the time of admission showed pathological Q waves in leads V1-V6. A transthoracic echocardiogram revealed left ventricular inferior and inferoseptal segment hypokinesis with reduced global ventricular function (EF Simpson, Bi-plane <40\%). The laboratory results indicated an elevated cardiac Troponin $\mathrm{T}(25 \mathrm{pg} / \mathrm{ml})$, which confirmed the development of acute myocardial infarction.

He was consulted for an emergency PCI. Selective coronary angiography revealed a 50\%-60\% narrowing lesion in the proximal segment of LAD and no evidence of other atherosclerosis in LCx and RCA. A subsequent angiography of the LMCA was undertaken and showed a large, tortuous septal branch originating from the proximal segment of LAD. At the terminal end of this artery, a small volume of contrast agents went out of the coronary artery (Figure 1), but it was impossible to determine the exact drainage site.
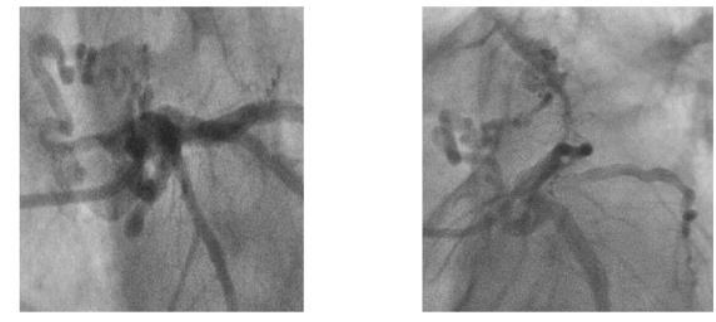

Figure 1: Coronary angiogram image showing fistula from left coronary artery

These findings showed that an atherosclerosis lesion in LAD was not the culprit lesion, and the diagnosis of coronary fistula-induced myocardial infarction due to the "steal phenomenon" was established. Owing to the complex anatomy, PCI did not clearly define the distal course and drainage 
of the CAF, and the physician decided to stop the intervention at that time. A contrast - enhanced computed tomography angiography (CTA) was subsequently employed to investigate the course and anatomy of it. The CTA result was assessed using a window width and level of $300 / 50$ Hounsfield units and a slice thickness of $0.5 \mathrm{~mm}$. The findings highly suggest a large communication lesion between LCM and the anterior side of the main pulmonary artery with the size $3.6 * 2 \mathrm{~mm}$ (Figure 2).

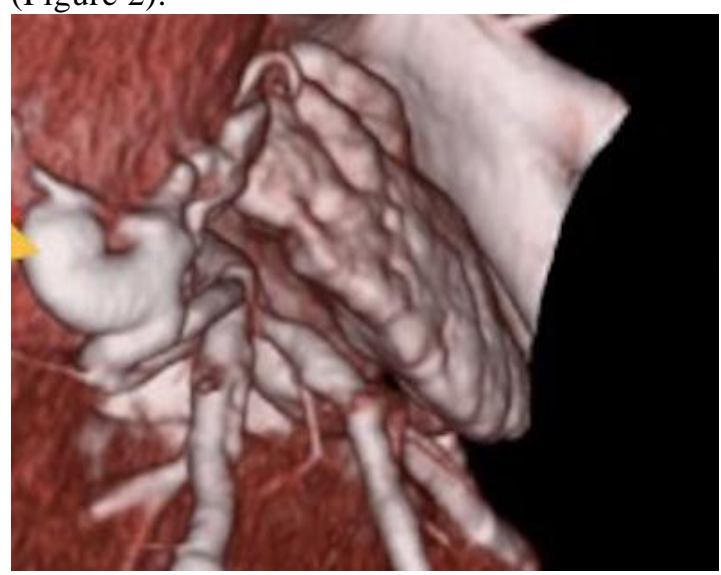

Figure 2: Coronary Computed Tomography Angiography image showing fistula (yellow arrow) from LMCA to pulmonary artery

A follow-up elective PCI has been consulted to tackle the CAF and atherosclerosis lesion in LAD. During the intervention, the lesion in LAD was dilated, and an Everolimus-eluting stent $(3 \times 33 \mathrm{~mm}$, Xience Prime, Abbott) was successfully deployed over the lesions in LAD to cover over the origin of CAF without obstructing any vessels nearby. After the procedure, a TIMI 3 flow in the left coronary artery and occlusion of the CAF were confirmed. After that, the patient was transferred to the CCU in stable condition $\mathrm{T}$, and dual antiplatelet therapy (aspirin and ticagrelor) in addition to anti-ischemic medications were employed. A week later, a TTE examination was conducted and showed no signs of segmental wall-motion abnormalities nor shunted flow, with global ventricular ejection fraction significantly improved (EF Simpson, Bi-plane = $55 \%$ ). He was discharged in a stable condition with optimal pharmacological treatments and has been closely followed up in our Outpatient Department. At 1-month follow-up in the clinic, he reported complete resolution of his symptoms, and the LV contractile function remained stable

\section{DISCUSSION}

Coronary artery fistula (CAF) refers to anomalous coronary artery drainage to a cardiac chamber or other vessels and the coronary vein sinus. Although it is often due to congenital anomalies, acquired fistulae have been reported in trauma cases from invasive procedures such as endomyocardial biopsy or coronary artery bypass surgery, and in some cases of Takayasu's arteritis. ${ }^{1}$ Differential diagnosis includes a wide range of heart diseases: systemic or pulmonary arteriovenous fistula, patent ductus arteriosus, aortopulmonary window, or ruptured sinus of Valsalva aneurysm.

The most significant pathophysiology consequence in the patient with CAF is the "steal" phenomenon, in that coronary artery blood flow is diverted away from the high resistance zone in the myocardial capillaries to the low resistance zone of the anomalous channel (cardiac chambers, pulmonary arteries), which results in the reduction of the distal coronary blood flow. Clinical complications depend on the fistula's size, the pressure gradient between the terminals of fistulae, and the volume of the shunt flow. ${ }^{2}$ Most of the fistulae do not result in significant clinical consequences, but medium to large size CAF can lead to volume overload, and pulmonary hypertension has been reported. Angina, dyspnea on exertion, syncope, and palpitations are other reported symptoms. ${ }^{3}$ Acute coronary syndrome in cases of coronary artery fistula is rare. For our patient, the steal syndrome accompanied a significant atherosclerosis lesion that formed conditions suitable for acute myocardial infarction. The diagnosis of coronary artery fistulas relies primarily on clinical manifestations and imaging modalities. Two-dimensional and Colour Doppler cardiac ultrasound can show the coronary artery's origin and course and abnormal angioectasias.

CMR imaging and CTA are non-invasive and accurate imaging modalities that positively detect a differential diagnosis of coronary artery fistula. Many studies have shown that CTA is more efficient in detecting coronary artery anomalies than traditional coronary angiography. ${ }^{4}$ However, coronary artery fistulae are usually diagnosed incidentally during cardiac catheterization. Coronary artery angiography can directly reveal the blood vessel distribution and the fistula drainage situation, which is essential information for diagnosis and treatment decisions. $^{5}$

Currently, there is no consensus for the treatment of patients with CAF. Asymptomatic patients who have small fistulae can be treated conservatively. In patients with symptomatic fistula or complications, cardiac intervention (surgical ligation or transcatheter closure (TCC)) should be mainly performed as early as possible. Percutaneous TCC is the preferred method: it is less invasive and avoids the potential complications of surgery. TCC may not be conducted in a tortuous or in a large fistula. In these cases, surgical closure should be considered. ${ }^{6}$ Elective closure of a fistula is recommended in children older than 3 to 5 years of age as they are at increased risk of complications. Surgical ligation should 
be considered to avoid aneurysm formation and rupture with the fistulae arising from the proximal segment of coronary arteries. $^{3}$

In summary, a coronary artery fistula is a rare congenital or acquired coronary artery anomaly. Patients may be symptomatic or asymptomatic due to the severity of the steal phenomenon. Although coronary angiography remains the primary diagnosis method, advanced imaging modalities such as multidetector computed tomography provide more complementary data about fistula than a traditional coronary angiogram. There is no consensus for the treatment of patients with CAF. The surgical or percutaneous procedure can be considered for symptomatic patients with large fistula with high flow rates. Transcatheter closure of the fistula is the preferred treatment due to its efficacy and low risk of complication.

\section{AUTHORS' CONTRIBUTION}

QV: Concept and design, data acquisition, interpretation, drafting, final approval, and agree to be accountable for all aspects of the work. HV, TD: Data acquisition, interpretation, drafting, final approval and agree to be accountable for all aspects of the work.

Conflict of interest: Authors declared no conflict of interest.

\section{REFERENCES}

1. Raufi MA, Baig AS. Coronary artery fistulae. Rev Cardiovasc Med. 2014;15(2):152-7.

2. Hobbs RE, Millit HD, Raghavan PV, Moodie DS, Sheldon WC. Coronary artery fistulae: a 10-year review. Cleve Clin Q. 1982;49(4):191-7.

3. Challoumas D, Pericleous A, Dimitrakaki IA, Danelatos C, Dimitrakakis G. Coronary arteriovenous fistulae: a review. Int J Angiol. 2014;23(1):1-10.

4. Lim JJ, Jung JI, Lee BY, Lee HG. Prevalence and types of coronary artery fistulas detected with coronary CT angiography. AJR Am J Roentgenol. 2014;203(3):W237-43.

5. Varghese A, Keegan J, Pennell DJ. Cardiovascular magnetic resonance of anomalous coronary arteries. Coron Artery Dis. 2005;16(6):355-64.

6. Armsby LR, Keane JF, Sherwood MC, Forbess JM, Perry SB, Lock JE. Management of coronary artery fistulae. Patient selection and results of transcatheter closure. J Am Coll Cardiol. 2002;39(6):1026-32.

\section{Address for Correspondence:}

Dr. Quan Vo, University of Medicine and Pharmacy, Ho Chi Minh City, Ho Chi Minh, 70000, Vietnam.

Email: dr.duyquan@gmail.com 